\title{
Sustainable Development: Actual Trends on Synthetic Indicators, Non-aggregative and Configurational Approaches
}

\author{
Norat Roig-Tierno ${ }^{1} \cdot$ Alberto Arcagni $^{2}$ \\ Published online: 25 February 2021 \\ (c) The Author(s), under exclusive licence to Springer Nature B.V. part of Springer Nature 2021
}

\begin{abstract}
Sustainable development is key for the fundamental challenges of humanity. The use of non-aggregative approaches can be attractive when trying to understand the relationships of humanity with both nature and society. Qualitative Comparative Analysis (QCA) is a method that merges the advantages of qualitative and quantitative methodologies and identifies patterns of conditions that are necessary or sufficient for explaining an outcome. Partially ordered set (poset) theory is a branch of mathematics through which tools that allow dealing with multidimensional systems of ordinal data are obtained. Assessing well-being and development requires sharing a conceptual framework on its determinants, as well as on society, and needs from the identification of the most consistent and effective methodologies to build indicators that are easily understood by society. Sustainable development is an increasingly interesting issue whose academic development can be improved thank to the use of these methodologies, in its combination of environmental and socio-economic concerns.
\end{abstract}

Keywords Sustainable development · Qualitative comparative analysis (QCA) · FsQCA · Partially ordered set (poset)

\section{Introduction}

Due to its complexity, sustainable development has no unique definition; therefore, the construction of an indicator to measure the level of development reached by countries requires a detailed analysis of the determining variables.

For instance, in 2015, as part of the 2030 Agenda for Sustainable Development, UnitedNations defined a list of 17 goals, showed in Fig. 1 and described in United Nations (2021), each one containing different targets. Sustainable development is key to face the

Norat Roig-Tierno

norat.roig@upv.es

Alberto Arcagni

alberto.arcagni@uniroma1.it

1 Universitat Politècnica de València, Valencia, Spain

2 Sapienza University of Rome, Rome, Italy 


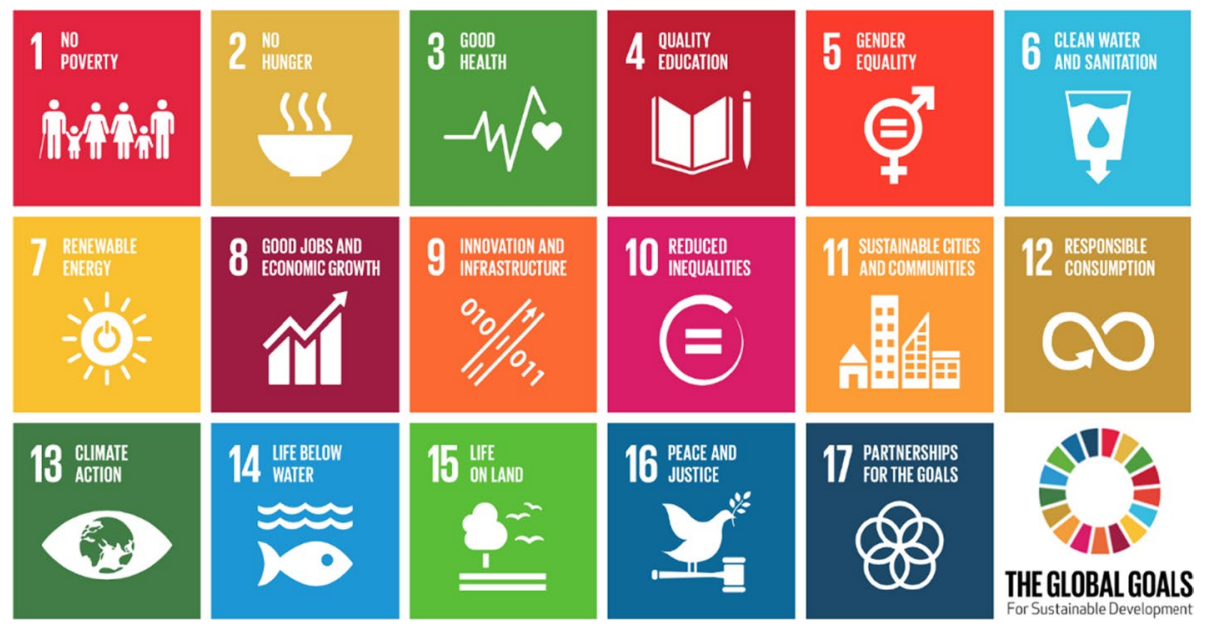

Fig. 1 Sustainable development goals. Note: this figure is licensed under CC BY-ND

fundamental challenges of humanity. The use of non-aggregative and configurational approaches can be useful when trying to understand the relationships between nature and society.

This special issue has three different objectives. Firstly, to analyze the new trends regarding synthetic indicators related to sustainable development. Secondly, to counteract the current trend of research on non-aggregative approaches that limits the appearance of new specific methodology proposals such as partially ordered set (poset) theory. Lastly, to identify paths that lead to a particular outcome using configurational methods such as Qualitative Comparative Analysis (QCA).

\section{The relevance of the methods}

To evaluate the relevance and importance of the certain methods, we will do an exploratory bibliometric analysis using the Web of Science (Sarin et al. 2020). Specifically, we will analyze the number of documents, the evolution of the citations and the overall number of citations in the "Core Collection Database" until 2020 that contain poset or QCA approaches. The results were retrieved in February 2021.

On the one hand, regarding the poset approach, the following search criteria was established: "partially ordered set" OR "partially ordered sets". As a result, we obtained 2.620 documents. However, the analysis was done over 2.352 articles. These articles had received 29.880 citations ( $h$ index $=64$ ). Figure 2 shows the number of publications per year and the accumulative number of citations. Note that the $52.89 \%$ of the articles were published in the last 10 years and more than $75 \%$ in the last 20 years (1.786 articles). These results show the increasing relevance of the method.

On the other hand, regarding the QCA methodology, the search criteria was "Qualitative Comparative Analysis". The search provided 1.978 articles from 2.180 documents with 26.329 citations ( $\mathrm{h}$ index $=66$ ). Figure 3 shows the evolution of publications and citations about QCA. Specifically, the $81.7 \%$ of the articles were done in the last 5 years (1.608 


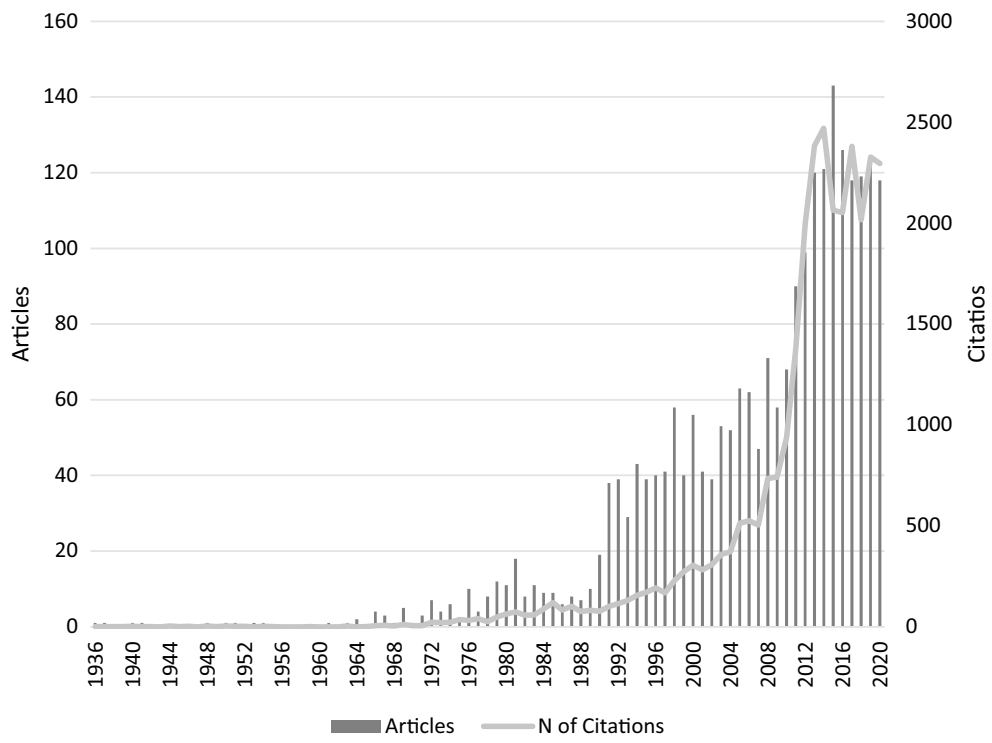

Fig. 2 Number of articles and citations per year in poset approach

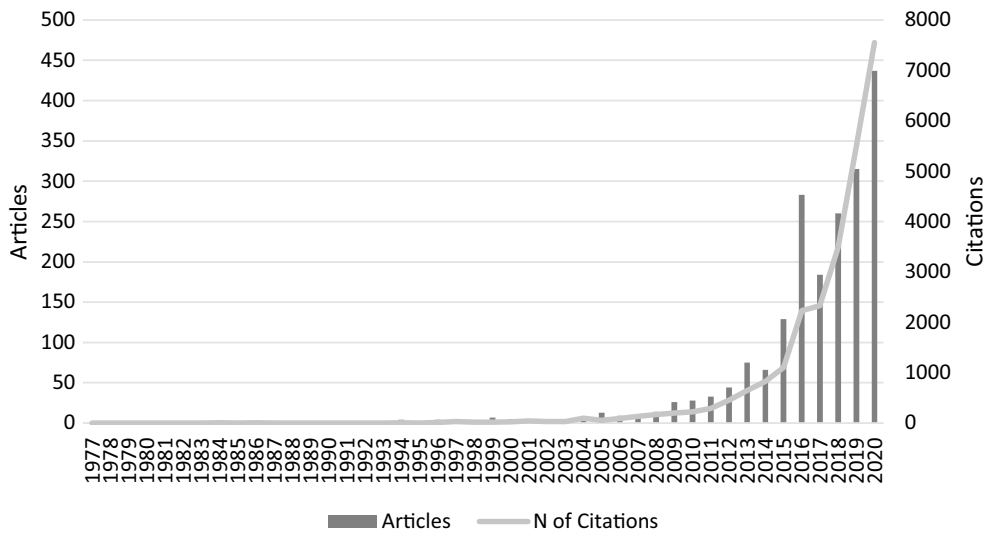

Fig. 3 Number of articles and citations per year in QCA approach

articles). Again, these results show that the use of this methodology is increasing its relevance and interest from the academia in the last few years.

In sum, Figs. 2 and 3 show the increasing attention that these methodologies are receiving from the academia.

\subsection{Poset: Partially Ordered Set}

It is possible to assign to each target one or more variables. Such variables may be quantitative, ordinal, or only binary (indicating deprivation or not). 
Once the variables are determined, we need to find a methodology to summarize them, to compare different situations and, if possible, to sort the results. Moreover, midway synthesis for each goal may be useful to analyze the policies of the Member States. But note that different natures and different topics characterize the previously defined variables, consequentially an indicator defined through an aggregative method may be not adequate.

Excluding the nominal scale, where modalities of variables cannot be sorted, the most general scale of measure is the ordinal one. Fattore (2016) determined that the mathematical representation of a multidimensional ordinal attribute is the partially ordered set (poset) and, in particular, the product order.

Now we report the definitions of poset and product order from Davey and Priestley (2002):

Let $P$ be a set. An order (or partial order) on $P$ is a binary relation $\leq$ on $P$ such that, for all $x, y, z \in P$ :

(i) $x \leq x$,

(ii) $x \leq y$ and $y \leq x$ imply $x=y$,

(iii) $x \leq y$ and $y \leq z$ imply $x \leq z$.

These conditions are referred to, respectively, as reflexivity, antisymmetry and transitivity. A set $P$ equipped with an order relation $\leq$ is said to be an ordered set (or partially ordered set).

Note that not necessarily all the elements of a poset may be comparable. Therefore, a poset is a definition that includes as particular cases the nominal scale (with no comparable modalities) and the ordinal scale (where all the modalities are comparable). A multidimensional ordinal attribute can be defined as the Cartesian product of its dimensions that are ordinal attributes i.e. ordered sets.

Let $P_{1}, \ldots, P_{n}$ be ordered sets. The Cartesian product $P_{1} \times \cdots \times P_{n}$ can be made into an ordered set by imposing the coordinatewise order defined by

$$
\left(x_{1}, \ldots, x_{n}\right) \leq\left(y_{1}, \ldots, y_{n}\right) \Longleftrightarrow(\forall i) x_{i} \leq y_{i} \text { in } \quad P_{i} .
$$

The product order is therefore an order relation that associated with the previous Cartesian product defines a poset.

Note that, generally, an ordered attribute has a finite set of modalities, but the posetic approach can be applied also to continuous variables (due to the hierarchy of scales). In this case, the sets are not finite, but it is possible to consider a finite subset of the multivariate attribute, as the observed profiles, that is still a poset. If the multivariate attribute is completely quantitative, it may still make sense to apply the posetic approach to define non-aggregative indicators.

Once the data structure is defined, it is possible to apply many different techniques to describe and summarize the information contained in the observations. Some of these techniques are described, discussed, and applied in the articles of this special issue.

\subsection{QCA: Qualitative Comparative Analysis}

Ragin $(2000,2009,2014)$ developed a comparative protocol to analyze causal configurations in a systematic way. This protocol is the Qualitative Comparative Analysis or QCA that can be implemented with different nuances (Schneider and Wagemann 2012) and which involves four analytical steps or phases: 
1. Build the comparative table (or data matrix) to organize the qualitative information and identify possible necessary conditions from the similarity method;

2. Articulate the typological theory and classify the cases according to this typology. The analysis of this "truth table" allows identifying sufficient causal configurations to generate the results or configurations;

3. Analyze the counterfactuals; those configurations for which we do not have examples in the data;

4. Reduce the number of sufficient configurations (when possible) through a process of logical minimization.

QCA is an analytical technique that combines quantitative and qualitative methodology (Ragin, 12,000; 2009, 2014). This technique originally focused on small samples, but its subsequent development allows its application to broader contexts (Garcia-Alvarez-Coque et al. 2021).

QCA applications were initially focused on case studies. The drawback of these methods is the impossibility of generalizing the results to other similar cases. However, QCA is currently based on case studies and on the analysis of empirical data for the generalization of the analysis, considering their possible replication in later studies, and constructing logical propositions as a result of the qualitative study of the phenomenon in question (NietoAleman et al. 2019).

QCA is a comparative methodology that allows to explore complex causality patterns solving the problem of sample size (Rioux and Ragin 2009). Specifically, QCA examines the causal and independent variables, and how the combination between them causes the same result. In general, QCA departs from the assumptions of uniformity and symmetry.

\section{Contributions}

The contribution by Garcia-Alvarez-Coque, Roig-Tierno, Sanchez-Garcia and Mas-Verdu is entitled 'Knowledge Drivers, Business Collaboration and Competitiveness in Rural and Urban Regions' and deals with the innovation system and its contribution to regional development, distinguishing between rural and urban regions. The regional unit for the application of the QCA was the Nomenclature of Territorial Units for Statistics level 2 (NUTS 2) for European regions and 256 regions were analyzed. The results of this study highlight that territorial innovation policies arise from a mix of business collaboration, university excellence, and public and private R\&D.

The contribution entitled 'What Type of Entrepreneurship Leads to Sustainable Development? A Configurational Approach' is written by Mas-Tur, Guijarro and Carrilero and aims to identify the characteristics of entrepreneurs that enhance sustainable development using Global Entrepreneurship Monitor (GEM) and Global Innovation Index (GII). Using QCA, the authors show that the characteristics of entrepreneurs are fundamental for the implementation of sustainability in the companies. Moreover, business model development can be seen as a bridge between business strategy and business practices that foster sustainability practices and that pay attention to the circular economy.

The contribution of Carlsen and Bruggemann, titled "Stakeholders' opinions. Food sustainability as an exemplary case", introduces the complexity of creating a composite indicator. They focus on the weighted sum of single indicators as the most used aggregation technique. This approach may appear simple but the choice of different structures of 
weights lead to different results that can modify the final ranking. They show the example of the Food Sustainably Index (FSI), composed by three main indicators, and evaluated for 34 countries. Assuming four stakeholders, they propose four different schemes of weights and evaluate the FSI. They use the different results to create a coordinatewise partial order and to compare the different rankings of the countries. This poset allows to observe which comparabilities are preserved and which not, due to the different opinions of the stakeholders. The paper shows the limits of the aggregative approaches and the next contributions apply some poset-based alternatives.

The paper "Targeting policies for multidimensional poverty and social fragility relief among migrants in Italy, using F-FOD analysis" by Rimoldi, Arcagni, Fattore and Barbiano di Belgiojoso applies the Fuzzy First-Order Dominance (F-FOD) methodology to rank migrant populations in Lombardy (Italy). The F-FOD is a statistical procedure for the comparison of frequency distributions on systems of ordinal indicators. It returns a matrix indicating the degree of comparability for each pair of distributions. Through this matrix, it is possible to evaluate dominance and incomparability scores. The first scores are used to rank the distributions and the second to control the degree of uncertainty. In this paper, the methodology is applied on indicators about poverty and social fragility and compares distributions of migrants distinguished by country of origin and time since migration.

The contribution "Synthesis of multi-indicator system over time: a poset-based approach" by Alaimo, Arcagni, Fattore and Maggino, proposes a methodology that start from a numerical multi-indicator system and, instead of applying an aggregative approach, creates a coordinatewise partial order of the observations to create a synthesis. Then it uses the poset to synthetize the multi-indicator system. This methodology is applied the Italian regions data about the first of the Sustainable Development Goals. The evolution of the results is studied over time and compared with the Adjusted Mazziotta-Pareto Index.

Alaimo, Ciacci and Ivaldi suggest in the paper "Measuring sustainable development by non-aggregative approach" apply the poset-based approach on data from the European Foundation for the Improvement of Living and Working Conditions (Eurofound). They provide synthetic measures about the sustainable development in the European countries and test the validity and consistency of the results comparing them with the most common aggregative methods.

In the contribution "Analysis of sustainability propensity of bike-sharing customers using Partially Ordered Sets methodology" Maranzano, Ascari, Chiodini and Manzi created two posets starting from ordinal variables obtained from a survey performed among the subscribers of the bike-sharing system in Milan, Italy. The two posets are used to get scores about the green propensity of the subscribers and their satisfaction with the service and the authors analyze the relation between the two variables.

In "Between social sustainability and subjective wellbeing. The role of decent work" Conigliaro introduces the concept of sustainability in relation to decent work, then she uses posets in a two-step synthesis about the achievement of sustainable development targets in terms of decent work in European Union countries.

\section{References}

Davey, B. A., \& Priestley, H. A. (2002). Introduction to lattices and order. Cambridge University Press.

Fattore, M. (2016). Partially ordered sets and the measurement of multidimensional ordinal deprivation. Social Indicators Research, 128(2), 835-858. 
Garcia-Alvarez-Coque, J. M., Mas-Verdú, F., \& Roig-Tierno, N. (2021). Life below excellence: exploring the links between top-ranked universities and regional competitiveness. Studies in Higher Education, 46(2), 369-384.

Nieto-Aleman, P. A., Garcia-Alvarez-Coque, J. M., Roig-Tierno, N., \& Mas-Verdú, F. (2019). Factors of regional poverty reduction in Colombia: Do institutional conditions matter? Social Policy \& Administration, 53(7), 1045-1063.

Ragin, C. C. (2000). Fuzzy-set social science. University of Chicago Press.

Ragin, C. C. (2009). Redesigning social inquiry: Fuzzy sets and beyond. University of Chicago Press.

Ragin, C. C. (2014). The comparative method: Moving beyond qualitative and quantitative strategies. University of California Press.

Sarin, S., Haon, C., Belkhouja, M., Mas-Tur, A., Roig-Tierno, N., Sego, T., Porte, A., Merigó, J., \& Carley, S. (2020). Uncovering the knowledge flows and intellectual structures of research in technological forecasting and social change: A journey through history. Technological Forecasting and Social Change, 160, 120210.

Schneider, C. Q., \& Wagemann, C. (2012). Set-theoretic methods for the social sciences: A guide to qualitative comparative analysis. Cambridge University Press.

United nations sustainable development - 17 goals to transform our world. (2021). Retrieved February 12, 2021, from https://www.un.org/sustainabledevelopment

Publisher's Note Springer Nature remains neutral with regard to jurisdictional claims in published maps and institutional affiliations. 